\title{
Incremento de la rentabilidad económica en el cultivo de papa criolla mediante fertilización con manganeso
}

\section{Increased profitability of the yellow potato crop by fertilization with manganese}
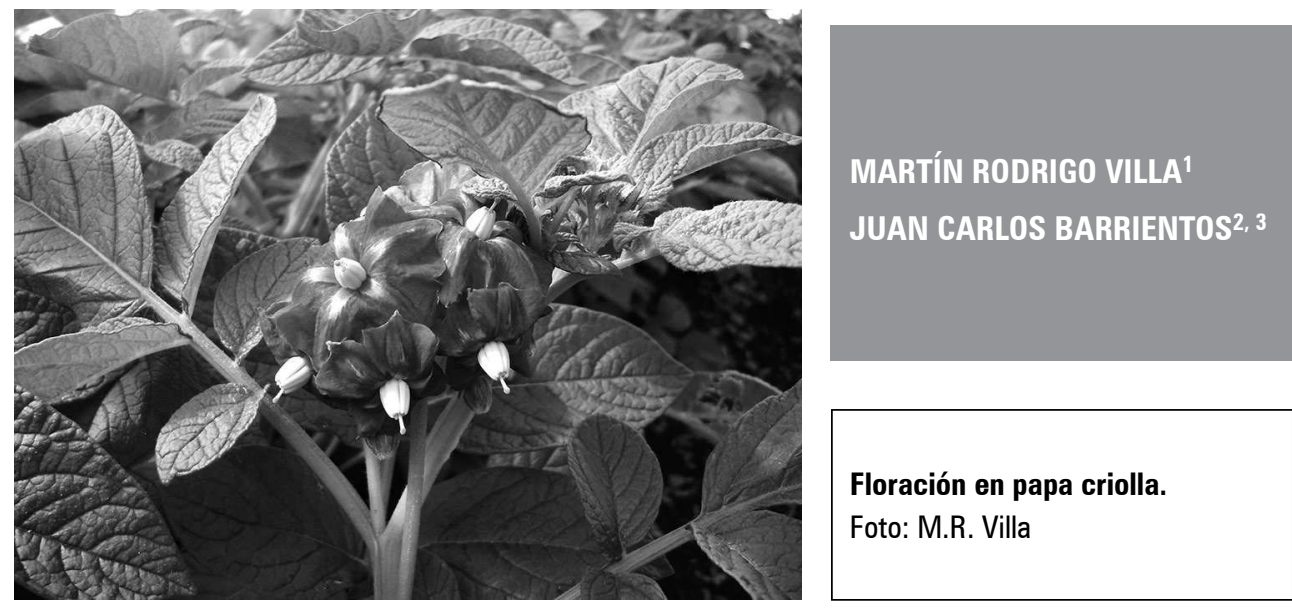

\section{RESUMEN}

El manganeso (Mn) es importante para la fotosíntesis y formación de carbohidratos solubles en la planta. La adición de $\mathrm{Mn}$ en la fertilización del cultivo de papa en Colombia no es común. Sin embargo, hay trabajos que demuestran que una adición de $\mathrm{Mn}$ tiene efectos favorables en la producción y calidad del producto, sobre todo en zonas con bajos contenidos nativos de este nutriente. Una mejora en la producción tiene que ver también con una mejora en la rentabilidad. Bajo este escenario, el presente estudio se propuso determinar de manera experimental el incremento de rentabilidad por adición de $\mathrm{Mn}$ en la fertilización de papa criolla en Colombia. El ensayo se realizó el primer semestre del 2008 en el municipio El Rosal, Cundinamarca, adicionando $2 \mathrm{~kg} \mathrm{ha}^{-1}$ de $\mathrm{Mn}$ (sulfato de $\mathrm{Mn}$ ) en la fertilización. Los resultados muestran que la aplicación de $\mathrm{Mn}$ al cultivo de papa variedad Criolla incrementó el rendimiento en 52\%, así como el porcentaje (de $42 \%$ a $58 \%$ ) de la producción de la categoría (calidad) primera. Los ingresos se incrementaron de manera similar al rendimiento (58\%). Los costos totales de producción/ha aumentaron en $11 \%$, pero el costo unitario disminuyó en $27 \%$. Finalmente, los resultados muestran un incremento de la rentabilidad de $19 \%$ a $69 \%$. La aplicación edáfica de $\mathrm{Mn}$ (sulfato de $\mathrm{Mn}$ ) es una opción viable para el manejo nutricional del cultivo de papa variedad Criolla Colombia, y muy favorable económicamente.

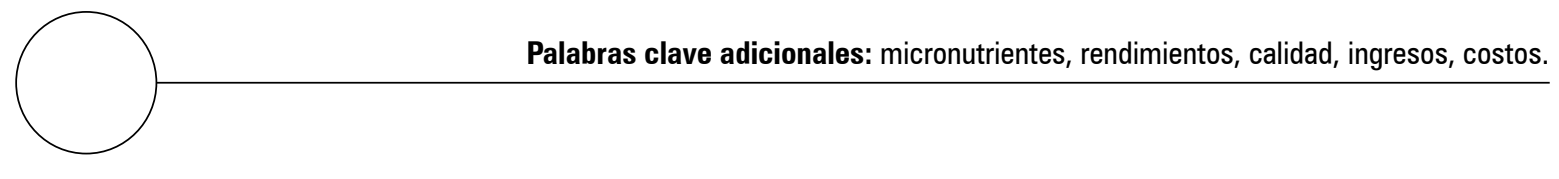

Facultad de Agronomía, Universidad Nacional de Colombia, Bogotá (Colombia).

Facultad de Agronomía, Departamento de Agronomía, Universidad Nacional de Colombia, Bogotá (Colombia).

Autor para correspondencia. jcbarrientosf@unal.edu.co 


\section{ABSTRACT}

Manganese $(\mathrm{Mn})$ is important in photosynthesis and formation of soluble carbohydrates in the plant. The addition of $\mathrm{Mn}$ in the fertilization of potato cultivation in Colombia is not common. However, studies show that adding $\mathrm{Mn}$ to crops has favorable effects on production and product quality, especially in areas with low levels of this nutrient. Improved production can also mean improved profitability. Under this scenario, this paper aimed to experimentally determine increased profitability by adding $\mathrm{Mn}$ in the fertilization of the yellow potato in Colombia. This study was conducted in the first half of 2008 in the municipality of El Rosal, Cundinamarca; adding $2 \mathrm{~kg} \mathrm{ha}^{-1}$ of $\mathrm{Mn}$ (Mn sulfate) to the fertilization. The results show that the application of $\mathrm{Mn}$ in yellow potato crops increases yield by $52 \%$ and percentage (from $42 \%$ to $58 \%$ ) of first quality products. Revenues increased similarly to yield (58\%). Total production costs/ha increased by $11 \%$, but the unit cost decreased by $27 \%$. Finally, the results show an increase in profitability from $19 \%$ to $69 \%$. The application of $\mathrm{Mn}$ (Mn sulfate) is a viable option for the nutritional management of the yellow potato in Colombia, and is also economically favorable.

Additional key words: micronutrients, yields, quality, revenue, costs.

Fecha de recepción: 02-02-2012

Aprobado para publicación: 29-05-2012

INTRODUCCIÓN

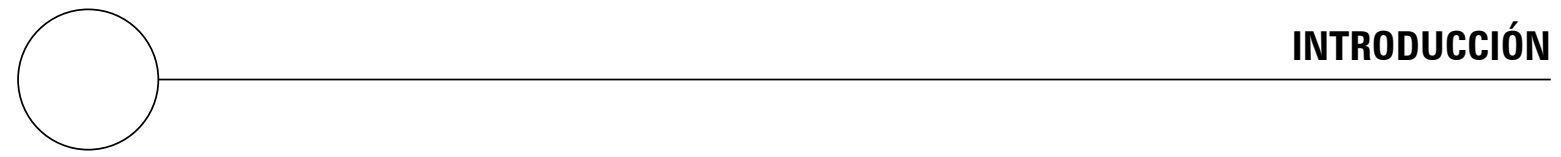

La papa criolla, tubérculo diploide, actualmente clasificada como Solanum tuberosum Grupo Andigena (Spooner et al., 2007), es un producto de mucha importancia en la economía campesina de las zonas paperas de Colombia, donde cubre aproximadamente 12.000 ha, ubicadas principalmente en los departamentos de Cundinamarca, Boyacá y Nariño (Martínez et al., 2006). Su cultivo se realiza bajo las siguientes condiciones agroecológicas: temperatura promedio $10-20^{\circ} \mathrm{C}$, precipitación anual de $900 \mathrm{~mm}$, altitud entre 1.800 y $3.200 \mathrm{msnm}$ y suelos preferiblemente con textura franca (Pérez et al., 2008).

La papa criolla es parte importante de la culinaria colombiana, se destaca por su valor nutritivo, su contenido de carbohidratos (19,4\%), minerales (Fe, Zn, Cu y Ca), carotenoides (Bonierbale et al., 2004), vitaminas A, C y complejo B, fibra cruda $(0,6 \%)$, alto contenido de materia seca $(22,8 \%)$, baja concentración de grasa $(0,1 \%)$ y un contenido de proteí- nas que varía entre 0,7 a 4,6\% (FAO, 1998; Moreno, 2000; Alvarado, 2005; MADR, 2006a).

E1 $98 \%$ de las unidades productivas de papa en Cundinamarca corresponden a pequeños y medianos agricultores, $74 \%$ entre $500 \mathrm{~m}^{2}$ y 1 ha, y $24 \%$ entre 1 y 3 ha; esa misma tendencia se puede ver en los departamentos de Boyacá y Nariño, cifras que le otorgan a este cultivo un carácter minifundista (Nustez, 2008). Además, las unidades de producción mencionadas se caracterizan por tener un nivel tecnológico entre bajo a medio, y no disponen de recursos financieros para mejorar la productividad (Martínez et al., 2006). Aparte de generar empleo e ingresos para los productores, la papa criolla es uno de los cultivos promisorios y un producto exportable (MADR, 2006b). Para cumplir el propósito exportador es necesario mejorar los rendimientos y la calidad del producto, así como disminuir los costos unitarios de producción. En este último 
aspecto se hace imperativo el uso eficiente de los insumos, especialmente de los fertilizantes (Martínez et al., 2006).

La función más importante del manganeso es la relacionada con la fotólisis del agua y con el sistema de evolución de $\mathrm{O}_{2}$ de la fotosíntesis que ocurre en los cloroplastos y es conocida como reacción de Hill (Marschner, 2006). La interrupción de esta reacción afecta el transporte de electrones, produciendo una disminución de la fotosíntesis y una desorganización del sistema de membranas tilacoides, las que contienen las enzimas que controlan la fotosíntesis (Salisbury y Ross, 2000; Clavijo, 2001; Taiz y Zeiger, 2006; Marschner, 2006; Kirkby y Römheld, 2007). El Mn es importante en el mantenimiento de las biomembranas y del aparato fotosintético al proteger los tejidos del efecto deletéreo de los radicales libres de oxígeno (superóxido $=\mathrm{O}_{2}^{-}$) (Marschner, 2006; Kirkby y Römheld, 2007). Una leve deficiencia de Mn afecta la fotosíntesis y reduce el nivel de carbohidratos solubles en la planta; una deficiencia más severa de $\mathrm{Mn}$ rompe la estructura de los cloroplastos, siendo esta una condición irreversible (Kirkby y Römheld, 2007).

El requerimiento de $\mathrm{Mn}$ en los suelos del cultivo de papa es de aproximadamente $50 \mathrm{mg} \mathrm{L}^{-1}$, con un rango de 25 a $300 \mathrm{mg} \mathrm{L}^{-1}$ (Ulrich, 1996; Bennett, 1996; Shuman, 2000; Salisbury y Ross, 2000). En el caso de este nutriente es común que se presenten más problemas por deficiencia que por toxicidad (Shuman, 2000; Matamoros, 2003). La aplicación de $\mathrm{Mn}$, aunque poco común en el cultivo de papa en Colombia, puede realizarse en forma de sulfato de manganeso $\left(\mathrm{MnSO}_{4} \cdot 4 \mathrm{H}_{2} \mathrm{O}\right)$ vía edáfica (Kirkby y Römheld, 2007).

No obstante, en la fertilización con Mn se deben considerar relaciones de sinergismo o de antagonismo, principalmente con $\mathrm{N}$ y Fe (Shuman, 2000; León, 2001; Fageria et al., 2007). Gómez (2005) establece que en suelos paperos de $\mathrm{Na}$ riño, Cundinamarca y Boyacá, una relación Fe/
Mn mayor a 15 puede provocar una deficiencia de $\mathrm{Mn}$; mientras que una relación menor a 10 induce deficiencia de Fe. Según Marschner (2006), los síntomas de deficiencia de Mn se presentan en las partes jóvenes de la planta, debido a que es un elemento inmóvil en el floema. Estos síntomas se manifiestan en la variedad Criolla Colombia a través de un color bronce del cogollo (Ulrich, 1996). Esta coloración es muchas veces tomada equivocadamente como una característica varietal de la papa Criolla Colombia (L.E. Rodríguez, comunicación personal).

De acuerdo al Consejo Nacional de la Papa (2010), los costos de producción de 1 ha de papa criolla para el año 2008 se situaron en \$8.307.327. De acuerdo con esta misma fuente, el precio promedio de venta a nivel de productor estaba en $\$ 751$ / $\mathrm{kg}$. Si se considera un rendimiento promedio de $13 \mathrm{t} \mathrm{ha}^{-1}$, entonces se tiene una rentabilidad aproximada de $18 \%$, y un costo unitario de $\$ 639$ / $\mathrm{kg}$. Esta información se constituye en referencia para los análisis económicos aquí desarrollados.

De manera general, la actual práctica de fertilización del cultivo de papa en Colombia no contempla la aplicación de micronutrientes, en especial de $\mathrm{Mn}$; hecho que subestima su importancia, más aún si se considera que es un micronutriente con bajos contenidos nativos en el suelo (Gómez et al., 2006). Los resultados de investigación encontrados por Gómez et al. (2006) en Solanum tuberosum L. y Pisum sativum L. y por Pérez et al. (2008) en papa criolla, dan cuenta de las bondades productivas de la aplicación de $\mathrm{Mn}$ en los cultivos mencionados.

Partiendo de la hipótesis de que la adición de Mn en la fertilización del cultivo de papa criolla Colombia en Cundinamarca posibilita un incremento de su rendimiento y una mejora de su calidad, en consecuencia mayores beneficios económicos para los productores, se plantea como objetivo determinar, de manera experimental, el incremento de rentabilidad del cultivo por efecto de esta práctica. 


\section{MÉTODO DE INVESTIGACIÓN}

\section{Lugar y momento de realización del experimento}

Esta investigación se basa en información empírica que se deriva de ensayos en campo. El trabajo experimental se realizó durante el primer semestre del año 2008 en la finca San Gabriel en el municipio El Rosal en el departamento Cundinamarca, ubicada a una altitud de 2.685 $\mathrm{msnm}$, con una precipitación anual de $825 \mathrm{~mm}$, $81 \%$ de humedad relativa y una temperatura promedio de $13^{\circ} \mathrm{C}$.

\section{Características del material vegetal}

Las semillas utilizadas fueron tubérculos de diámetro entre 2 y $4 \mathrm{~cm}$ de la variedad Criolla Colombia. Esta variedad se caracteriza por su crecimiento erecto, buen desarrollo de follaje, flor de color lila oscuro, tubérculos de forma redonda, puntos de brote semiprofundos, piel y pulpa de color amarillo intenso, ciclo corto (120 d en promedio), ausencia de periodo de reposo y rendimiento promedio de 13 a $15 \mathrm{t} \mathrm{ha}^{-1}$ (Rodríguez et al., 2009).

\section{Forma de aplicación del manganeso}

Como fuente del nutriente se utilizó Microman ${ }^{\circledR}$ (sulfato de $\mathrm{Mn}$, granulado al $20 \%$ ), el cual se aplicó en banda junto con los demás fertilizantes en el momento de la siembra. Este manejo se realizó como complemento al plan de fertilización convencional basado en análisis químico de suelos.

\section{Diseño experimental}

Los ensayos fueron distribuidos en campo en bloques completos al azar con cuatro repeticiones, en los cuales, $0 \mathrm{~kg} \mathrm{ha}^{-1}$ de $\mathrm{Mn}$ corresponde al testigo y $2 \mathrm{~kg} \mathrm{ha}^{-1}$ de $\mathrm{Mn}$ (sulfato de $\mathrm{Mn}$ ) a la dosis del micronutriente recomendada para esta varie- dad (Villa et al., 2011). El área experimental total constó de 172,8 $\mathrm{m}^{2}$ ubicada dentro de un cultivo (0,9 $\mathrm{m}$ entre surcos por $0,3 \mathrm{~m}$ entre plantas) y una densidad de 37.000 plantas/ha.

\section{Información de campo y análisis de la información}

Inmediatamente después de la cosecha (105 días después de la siembra) en el mes de junio, la producción se clasificó por calidades en: categoría primera (diámetro $>4 \mathrm{~cm}$ ) y categoría segunda (diámetro entre 2 y $4 \mathrm{~cm}$ ). La cantidad de tubérculos con diámetros menores a $2 \mathrm{~cm}$ fue insignificante, por lo que no se la tuvo en cuenta para los registros. La información que se consideró para el análisis fue: rendimiento $\left(\mathrm{kg} \mathrm{ha}^{-1}\right)$ total y por categoría (calidad), precio de venta por calidades $(\$ / \mathrm{kg})$ y costos de producción $(\$ / \mathrm{ha})$. Para los cálculos también se incluyó datos de precios de Corabastos (Central Mayorista de Bogotá) y del Sistema de Información de Precios del Sector Agropecuario (Sipsa, 2008) reportados para el mes de junio. Se encontró un margen de intermediación de $18,2 \%$ y $30,5 \%$ en la categoría primera y segunda, respectivamente. También se encontró una diferencia de precios por categorías, siendo el precio de venta de papa criolla sin lavar categoría primera $\$ 800 / \mathrm{kg}$ y para categoría segunda $\$ 650$ / $\mathrm{kg}$. Los costos adicionales por la aplicación de $\mathrm{Mn}$ incluyen los costos del producto, mano de obra para cosechar la producción adicional lograda por el uso de manganeso, el transporte y los empaques adicionales para su comercialización. Sin embargo, el costo de mano de obra para la aplicación del micronutriente se ha excluido ya que la adición de $\mathrm{Mn}$ al suelo se realiza en conjunto con la fertilización convencional y la baja dosis del fertilizante no conlleva a generar un costo adicional. En ese sentido se ha tomado como base un costo de producción para el año 2008 igual a $\$ 8.315 .000 /$ ha (sin incluir los costos por la adición de manganeso). Para determinar la utilidad neta de la producción de papa criolla con adición de Mn se seguirán las siguientes ecuaciones: 
Rentabilidad $=\frac{I-C}{C} \times 100$

$I=P \times Q$

Donde:

$I=$ ingresos $/$ ha $(\$ /$ ha $)$,

$C=$ costos $/$ ha $(\$ /$ ha $)$,

$P=$ precio del producto $(\$ / \mathrm{kg})$,

$Q=$ cantidad de producción $\left(\mathrm{kg} \mathrm{ha}^{-1}\right)$.

\section{RESULTADOS Y DISCUSIÓN}

\section{Rendimiento}

El tratamiento en el que se adicionó manganeso presenta un incremento del rendimiento que se encuentra alrededor del $50 \%$ con respecto al testigo (tabla 1), lo que indica que la aplicación de $\mathrm{Mn}$ ejerce una respuesta positiva en el rendimiento de papa variedad Criolla Colombia. Resultados similares se encontraron en investigaciones realizadas por Gómez et al., (2006) en papa variedades Diacol Capiro y Parda Pastusa en las cuales la aplicación de Mn incrementó entre $10 \%$ y $17 \%$ el rendimiento con respecto a la fertilización convencional (macronutrientes) en dos suelos de la Sabana cundiboyacense. Al realizar la prueba de comparación de promedios de Duncan $(\alpha=0,05)$, se presentaron diferencias significativas entre el testigo y el tratamiento con manganeso. Esta respuesta positiva se debe posiblemente a la alta relación Fe-Mn presente en el suelo, lo que limita la disponibilidad del Mn para la nutrición vegetal debido al antagonismo con el Fe (Shuman, 2000; Gómez et al., 2006; Fageria et al., 2007); de esta manera, la aplicación de Mn en dosis de $2 \mathrm{~kg} \mathrm{ha}^{-1}$ incrementa el contenido del micronutriente en el suelo y mejora así el balance Fe-Mn para finalmente producir mayor disponibilidad de Mn para el cultivo.

\section{Tabla 1. Rendimiento de papa criolla con y sin adición de Mn.}

\begin{tabular}{|c|c|c|}
\hline $\begin{array}{c}\text { Tratamiento } \\
\left(\mathrm{kg} \mathrm{ha}^{-1} \text { de } \mathrm{Mn}\right)\end{array}$ & $\begin{array}{c}\text { Rendimiento } \\
\left(\mathrm{kg} \mathrm{ha}^{-1}\right)\end{array}$ & $\begin{array}{c}\text { Variación del } \\
\text { rendimiento }(\%)\end{array}$ \\
\hline 0 & $13.900 \mathrm{~b}$ & 0 \\
\hline 2 & $21.200 \mathrm{a}$ & 52 \\
\hline
\end{tabular}

Promedios del rendimiento con letras distintas indican diferencia significativa según la prueba de Duncan $(P \leq 0,05)$.

\section{Calidad de tubérculos}

El tratamiento con manganeso presenta diferencias significativas en referencia al incremento en la producción de calidades "primera" con respecto al testigo, pasando la proporción del $58 \%$ al $42 \%$, respectivamente (tabla 2 ). El incremento de las "primeras" y la disminución de las "segundas" derivan en mayores ingresos para el agricultor ya que los precios para las primeras son mayores que para las segundas. La respuesta positiva en la categoría primera, se debe posiblemente al aumento en la síntesis de clorofila, metabolismo del $\mathrm{N}_{2}$ y transporte de electrones en la fotosíntesis (Marschner, 2006; Fageria et al., 2007; Kirkby y Römheld, 2007), lo que conduce al mejoramiento de la calidad del tubérculo cosechado. Resultados similares fueron encontrados por Gómez et al. (2007) en cebolla cabezona (Allium cepa), en los cuales se incrementó la producción de cebolla categoría primera bajo la aplicación de este micronutriente en Boyacá. Igualmente, se corrobora los resultados obtenidos por Pérez et al. (2008) en la variedad Criolla Colombia en la cual se incrementó el contenido de materia seca con la aplicación de $\mathrm{Mn}$ debido posiblemente a un aumento de la fotosíntesis neta.

\section{Ingresos económicos}

Los ingresos del tratamiento con manganeso presentan diferencias significativas con un incremento que se encuentra alrededor del 58\% respecto al testigo (tabla 3). El precio para la categoría primera es de $\$ 800 / \mathrm{kg}$ y para categoría segunda de $\$ 650 / \mathrm{kg}$, una diferencia de poco más del 20\%. El incremento del rendimiento, de la 
categoría primera y la superioridad del precio para esta determinan un significativo incremento de los ingresos como se observa en la siguiente tabla (tabla 3).

\section{Costos}

La adición de $\mathrm{Mn}$ genera un incremento de aproximadamente $11 \%$ (tabla 4) en los costos de producción por unidad de superficie. Esta diferencia se debe principalmente al incremento de rendimiento que implica una mayor demanda de mano de obra para cosecha y un gasto adicional en empaques, transporte al punto de comercialización y el costo del micronutriente.

Frente a los costos totales por unidad de superficie, los costos unitarios se comportan de otra manera, disminuyen con la adición de Mn (tabla $5)$. Y lo hacen en un porcentaje mayor al incremento de los costos totales. La gran ventaja de los costos unitarios frente a los costos de producción es que permiten compararlo con el precio de venta, y así saber inmediatamente si se va a ganar o perder en el negocio.

\section{Rentabilidad}

Se presentó diferencia estadística significativa de aproximadamente de $\$ 4.000 .000 /$ ha entre el tratamiento con $\mathrm{Mn}$ y el testigo (tabla 6). Con este incremento de la ganancia, la rentabilidad se incrementa en aproximadamente 3,5 veces, y esto con solo un incremento del $11 \%$ de los costos por hectárea.

\section{CONCLUSIONES}

La aplicación de manganeso al cultivo de papa variedad Criolla Colombia, bajo condiciones socioeconómicas y agroambientales del municipio de El Rosal (Cundinamarca), incrementa el rendimiento en 52\%. Adicionalmente, aplicar manganeso en la fertilización incrementa el porcentaje (de $42 \%$ a $58 \%$ ) de la producción de la categoría (calidad) primera, con la correspondiente disminución de la calidad segunda. Dado que la categoría primera recibe un precio mucho mayor (20\%) que la categoría segunda, se percibe un incremento de ingresos para el productor. Los in-

Tabla 2. Rendimiento por categorías de papa criolla con y sin adición de Mn.

\begin{tabular}{|c|c|c|c|c|c|}
\multirow{2}{*}{$\begin{array}{c}\text { Tratamiento } \\
\left(\mathrm{kg} \mathrm{ha}^{-1} \mathrm{de} \mathrm{Mn}\right)\end{array}$} & \multicolumn{3}{|c|}{ Rendimiento por categorías } & \multicolumn{2}{c|}{ Total } \\
\cline { 2 - 6 } & $\mathrm{kg} \mathrm{ha}^{-1}$ & $\%$ & $\mathrm{~kg} \mathrm{ha}^{-1}$ & $\%$ & $\mathrm{~kg} \mathrm{ha}^{-1}$ \\
\hline 0 & $5.800 \mathrm{~b}$ & 42 & $8.100 \mathrm{~b}$ & 58 & 13.900 \\
\hline 2 & $12.300 \mathrm{a}$ & 58 & $8.900 \mathrm{a}$ & 42 & 21.200 \\
\hline
\end{tabular}

Promedios del rendimiento con letras distintas indican diferencia significativa según la prueba de Duncan $(P \leq 0,05)$.

Tabla 3. Ingresos obtenidos por categorías con y sin adición de Mn.

\begin{tabular}{|c|c|c|c|c|c|c|}
\hline \multirow{3}{*}{$\begin{array}{c}\text { Tratamiento } \\
\text { (kg ha-1 de Mn) }\end{array}$} & \multicolumn{6}{|c|}{ Ingresos por categorías } \\
\hline & \multicolumn{2}{|c|}{ Primera $(\$ 800 / \mathrm{kg})$} & \multicolumn{2}{|c|}{ Segunda $(\$ 650 / \mathrm{kg})$} & \multicolumn{2}{|c|}{ Total } \\
\hline & $\begin{array}{l}\text { Rendimiento } \\
\left(\mathrm{kg} \mathrm{ha}^{-1}\right)\end{array}$ & $\begin{array}{l}\text { Ingresos } \\
\text { (\$/ha) }\end{array}$ & $\begin{array}{l}\text { Rendimiento } \\
\left(\mathrm{kg} \mathrm{ha}^{-1}\right)\end{array}$ & $\begin{array}{c}\text { Ingresos } \\
(\$ / \text { ha) }\end{array}$ & (\$/ha) & $\Delta \mathrm{I}^{1}(\%)$ \\
\hline 0 & $5.800 \mathrm{~b}$ & $4.640 .000 \mathrm{~b}$ & $8.100 b$ & $5.265 .000 \mathrm{~b}$ & $9.905 .000 \mathrm{~b}$ & 0 \\
\hline 2 & $12.300 \mathrm{a}$ & $9.840 .000 \mathrm{a}$ & $8.900 \mathrm{a}$ & $5.785 .000 \mathrm{a}$ & $15.625 .000 \mathrm{a}$ & 58 \\
\hline
\end{tabular}

${ }^{1}$ Variación en el incremento porcentual de ingresos.

Promedios con letras distintas indican diferencia significativa según la prueba de Duncan $(P \leq 0,05)$. 
Tabla 4. Costos de producción (\$/ha) con y sin adición de Mn.

\begin{tabular}{|c|c|c|c|c|c|c|c|}
\hline \multirow{3}{*}{$\begin{array}{c}\text { Tratamiento } \\
\text { (kg ha-1 }^{-1} \\
\text { de } \mathrm{Mn} \text { ) }\end{array}$} & \multicolumn{7}{|c|}{ Costos de producción } \\
\hline & \multirow{2}{*}{$\begin{array}{l}\text { Costo sin } \\
\text { adición de Mn }\end{array}$} & \multicolumn{6}{|c|}{ Costo adicional por fertilización con Mn } \\
\hline & & Costo del $\mathrm{Mn}$ & MOC1 & Empaque & Transporte & Costos totales & $\Delta \mathrm{C} 2(\%)$ \\
\hline 0 & 8.315 .000 & 0 & 0 & 0 & 0 & $8.315 .000 \mathrm{~b}$ & 0 \\
\hline 2 & 8.315 .000 & 35.000 & 420.000 & 295.000 & 108.500 & $9.233 .000 \mathrm{a}$ & 11 \\
\hline
\end{tabular}

${ }^{1}$ Mano de obra para cosechar la producción adicional lograda con aplicación de manganeso.

2 Incremento porcentual de los costos del tratamiento con Mn respecto al testigo.

Promedios de los costos totales con letras distintas indican diferencia significativa según la prueba de Duncan $(P \leq 0,05)$.

Tabla 5. Costos unitarios de producción con y sin adición de Mn.

\begin{tabular}{|c|c|c|c|c|}
\hline $\begin{array}{c}\text { Tratamiento } \\
\left(\mathrm{kg} \mathrm{ha}^{-1} \mathrm{de} \mathrm{Mn}\right)\end{array}$ & $\begin{array}{c}\text { Costos totales de } \\
\text { producción }(\$ / \mathrm{ha})\end{array}$ & $\begin{array}{c}\text { Rendimientos } \\
\left(\mathrm{kg} \mathrm{ha}^{-1}\right)\end{array}$ & $\begin{array}{c}\text { Costos unitarios de } \\
\text { producción }(\$ / \mathrm{kg})\end{array}$ & Variación (\%) \\
\hline 0 & 8.315 .000 & 13.900 & 598 & 0 \\
\hline 2 & 9.233 .000 & 21.200 & 436 & -27 \\
\hline
\end{tabular}

Tabla 6. Rentabilidad del cultivo con y sin adición de Mn.

\begin{tabular}{|c|c|c|c|c|}
\hline $\begin{array}{c}\text { Tratamiento } \\
\text { (kg ha-1 de } \mathrm{Mn} \text { ) }\end{array}$ & Ingresos (\$/ha) & Costos (\$/ha) & Ganancia (\$/ha) & Rentabilidad (\%) \\
\hline 0 & $9.905 .000 \mathrm{~b}$ & $8.315 .000 \mathrm{~b}$ & $1.590 .000 \mathrm{~b}$ & 19,1 \\
\hline 2 & $15.625 .000 \mathrm{a}$ & $9.233 .000 \mathrm{a}$ & $6.392 .000 \mathrm{a}$ & 69,2 \\
\hline
\end{tabular}

Promedios con letras distintas indican diferencia significativa según la prueba de Duncan $(P \leq 0,05)$.

gresos se incrementan de manera similar al rendimiento (58\%). Por otro lado, los costos totales de producción ( $\$ /$ ha) se incrementan en $11 \%$ por la adición de manganeso, debido principalmente al incremento de rendimiento que implica mayor demanda de mano de obra para cosecha y un gasto mayor en empaques y transporte del producto para su comercialización. Si embargo hay una disminución en un $27 \%$ del costo unitario.
Con un incremento en los rendimientos, un incremento en el porcentaje de producción de la categoría primera, así como con una disminución en los costos unitarios, se tiene un incremento de la rentabilidad de 3,5 veces frente al testigo. En resumen, la aplicación edáfica de $\mathrm{Mn}$ (sulfato de $\mathrm{Mn}$ ) es una opción viable para el manejo nutricional del cultivo de papa variedad Criolla Colombia, y muy favorable económicamente.

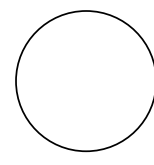

AGRADECIMIENTOS

Los autores expresan sus agradecimientos a Manuel Caicedo, Gerente de la Sociedad Agraria de Transformación de El Rosal, por su colaboración en la finca, y a la empresa Microfertisa S.A. por apoyar económicamente esta investigación. 


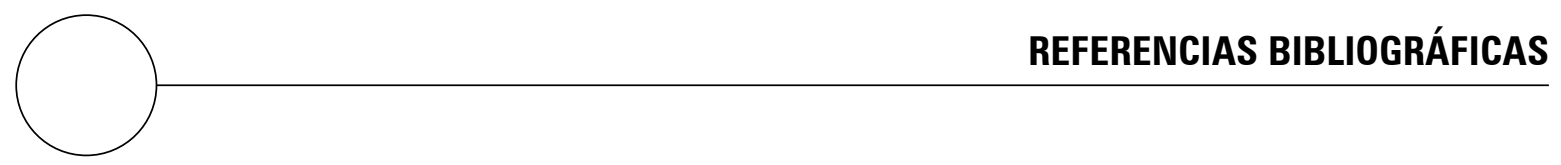

Alvarado, L. 2005. Principios de fisiología del cultivo de la papa. pp. 26-30. En: Memorias I Taller nacional sobre Suelos, Fisiología y Nutrición Vegetal en el Cultivo de la Papa. Centro Virtual de Investigación de la Cadena Agroalimentaria de la Papa (Cevipapa), Bogotá.

Bennett, W. 1996. Plant nutrient utilization and diagnostic plant symptoms. pp. 1-7. En: Bennett, W. F. (ed.). Nutrient deficiencies y toxicities in crop plants. The American Phytopathological Society, APS Press, St. Paul, MI.

Bonierbale, M., W. Amoros, J. Espinoza, E. Mihovilovich, W. Roca y R. Gómez. 2004. Recursos genéticos de la papa, don del pasado, legado para el futuro. Revista Latinoamericana de la Papa, Suplemento (Valdivia, Chile). pp. 3-14.

Clavijo, J. 2001. Metabolismo de los nutrientes en las plantas. pp. 11-28. En: Fertilidad de suelos, diagnóstico y control. $2^{\text {da }}$ ed. Sociedad Colombiana de la Ciencia del Suelo, Editorial Guadalupe Ltda., Bogotá.

Consejo Nacional de la Papa. 2010. Comparativo de precios al productor y precios al consumidor de papa variedad parda pastusa en Corabastos, 2008-2010. Documento Excel de uso interno. Bogotá.

Consejo Nacional de la Papa. 2010. Evolución de los costos totales de producción de papa Parda Pastusa, Diacol Capiro para industria y Papa Criolla en el altiplano cundiboyacense, 2002-2009. Documento Excel de uso interno. Bogotá.

Fageria, N., V. Baligar y R. Clark. 2007. Physiology of mineral nutrition. pp. 185-260. En: Physiology of crop production. Food Products Press, New York, NY.

FAO. 1998. Análisis de alimentos. Tubérculos, plátanos, almidones y azúcares. Food and Agriculture Organization of the United Nations (FAO), Roma e Instituto de Nutrición de Centro América y Panamá (INCAP). pp. 73-76.

Gómez, M.I. 2005. Guía técnica para el manejo nutricional de los cultivos: Diagnóstico, interpretación y recomendación de planes de fertilización. Microfertisa, Bogotá.

Gómez, M.I., M. López y C. Cifuentes. 2006. El manganeso como factor positivo en la producción de papa (Solanum tuberosum L.) y arveja (Pisum sativum L.) en suelos del altiplano cundiboyacense. Agron. Colomb. 24(2), 340-347.
Gómez, M.I., H. Castro, C. Gómez y F. Gutiérrez. 2007. Optimización de la producción y calidad en cebolla cabezona (Allium cepa) mediante el balance nutricional con magnesio y micronutrientes (B, Zn y Mn), Valle Alto del río Chicamocha, Boyacá. Agron. Colomb. 25(2), 339-348.

Kirkby, E.A. y V. Römheld. 2007. Micronutrientes na fisiología de plantas: Funcoes, absorcao e mobilidade. Encarte do Informacoes Agronómicas Brasil 118(2), 1-24.

León, L. 2001. Evaluación de la fertilidad del suelo. pp. 155-186. En: Fertilidad de suelos, diagnóstico y control. $2^{\text {da }}$ ed. Sociedad Colombiana de la Ciencia del Suelo, Editorial Guadalupe Ltda., Bogotá.

Marschner, H. 2006. Mineral nutrition of higher plants. $2^{\text {nd }}$ ed. Academic Press, New York, NY.

Martínez, H.; N. Pinzón y C. Barrios. 2006. La cadena de la papa en Colombia. Una mirada global de su estructura y dinámica 1991-2005. Documento de trabajo No. 100. Ministerio de Agricultura y Desarrollo Rural (MADR). Observatorio Agrocadenas Colombia e Instituto Interamericano de Cooperación Agropecuaria-IICA. Bogotá.

Matamoros, A. 2003. Suelos contaminados por metales traza. pp. 111-120. En: Manejo Integral de la Fertilidad del suelo. Sociedad Colombiana de Ciencia del Suelo. Bogotá.

Ministerio de Agricultura y Desarrollo Rural (MADR). 2006a. Perspectivas del sector agropecuario segundo semestre de 2006. Bogotá.

Ministerio de Agricultura y Desarrollo Rural (MADR). 2006b. Apuesta Exportadora Agropecuaria 20062020. MADR y Departamento Nacional de Planeación (DNP), Bogotá.

Moreno, J. 2000. Variedades de papa que se siembran en Colombia. Corporación Colombiana de Investigación Agropecuaria (Corpoica), C.I. Tibaitatá, Mosquera, Colombia.

Nustez, C. 2008. El cultivo de la papa en Colombia. En: Curso Sistemas de Producción. Facultad de Agronomía, Universidad Nacional de Colombia, Bogotá.

Pérez, L.C., L.E. Rodríguez y M.I. Gómez. 2008. Efecto del fraccionamiento de la fertilización con N, P, K y Mg y la aplicación de los micronutrientes $\mathrm{B}, \mathrm{Mn}$ y $\mathrm{Zn}$ en el rendimiento y calidad de papa criolla 
variedad Criolla Colombia. Agron. Colomb. 26(3), 477-485.

Rodríguez, L.E., C. Nustez y N. Estrada. 2009. Hoja divulgativa de la variedad Criolla Colombia. Facultad de Agronomía, Universidad Nacional de Colombia, Bogotá.

Salisbury, F. y W. Ross. 2000. Fisiología de las plantas 1. Células: agua, soluciones y superficies. Thomson Editores Spain Paraninfo, Madrid.

Shuman, L. 2000. Mineral nutrition. pp. 65-109. En: Baluška, F. (ed.). Plant-environment interactions. $2^{\text {nd }}$ ed. Marcel Dekker, New York, NY.

Sistema de Información de Precios del Sector Agropecuario (Sipsa). 2008. Boletín semanal de precios mayoristas 24(13). Bogotá.
Spooner, D., J. Núñez, G. Trujillo, M. del R. Herrera, F. Guzmán y M. Ghislain. 2007. Extensive simple sequence repeat genotyping of potato landraces supports a major reevaluation of their gene pool structure and classification. PNAS 104(49), 19398 19403

Taiz, L. y E. Zeiger. 2006. Plant physiology. $4^{\text {th }}$ ed. Sinauer Associates Publisher, Sunderland, MA.

Ulrich, A. 1996. Potato. pp. 149-155. En: Nutrient deficiencies y toxicities in crop plants. The American Phytopathological Society, APS Press, St. Paul, MI.

Villa, M.R., L.E. Rodríguez y M.I. Gómez. 2011. Effect of edaphic and foliar management of manganese on the yield of the Criolla Colombia cultivar. Agron. Colomb. 29 (3), 447-454. 